Supporting Information

\title{
Elucidating the Electronic Structure of High-Spin [Mn'II(TPP)Cl] Using Magnetic Circular Dichroism Spectroscopy
}

Mary Grace I. Galinato, ${ }^{1,2 *}$ Emily P. Brocious, ${ }^{2}$ Florian Paulat, ${ }^{1}$ Sherri Martin,,${ }^{1}$ Joshua Skodack, ${ }^{1}$ Jill B. Harland, ${ }^{1}$ Nicolai Lehnert ${ }^{1 *}$

${ }^{1}$ Department of Chemistry, University of Michigan, Ann Arbor, MI 48109-1055, USA. Email: lehnertn@umich.edu

2School of Science-Chemistry, Penn State Behrend, Erie, PA 16563, USA. Email: galinato@psu.edu

*Authors to whom correspondence should be addressed:

E-mail: lehnertn@umich.edu; galinato@psu.edu 


\section{Characterization of the $\left[\mathrm{Mn}^{\mathrm{III}}(\mathrm{TPP}) \mathrm{X}\right]$ complexes}

A. Room temperature UV-visible absorption features of [Mn $\left.{ }^{\mathrm{III}}(\mathrm{TPP}) \mathrm{X}\right]$ in $\mathrm{CH}_{2} \mathrm{Cl}_{2}$ in $\mathrm{nm}^{a}$

\begin{tabular}{|l|c|c|c|c|c|c|}
\hline \multirow{2}{*}{$\mathrm{X}$} & \multicolumn{2}{|c|}{ Soret } & \multicolumn{2}{c|}{ Q region } & \multicolumn{2}{c|}{ R ratio $^{b}$} \\
\cline { 2 - 7 } & Our work & Other groups & Our work & Other group & & Our work $^{d}$ Other group $^{d}$ \\
\hline $\mathrm{F}^{-}$ & 458 & $456 / 457$ & 572,606 & 572,606 & 4.50 & 4.16 \\
\hline $\mathrm{Cl}^{-}$ & 477 & $476 / 477$ & 578,618 & 583,619 & 1.96 & 2.02 \\
\hline $\mathrm{Br}^{-}$ & 486 & $484 / 486$ & 587,624 & 588,624 & 1.26 & 1.33 \\
\hline $\mathrm{I}^{-}$ & 498 & $496 / 498$ & 596,637 & 598,634 & 0.62 & 0.65 \\
\hline
\end{tabular}

${ }^{a}$ Only the band energies of [ $\left.\mathrm{Mn}^{\mathrm{III}}(\mathrm{TPP}) \mathrm{Cl}\right]$ were determined by Gaussian deconvolution. Therefore, there may be some discrepancies in other spectral features observed from our work relative to other groups (e.g. bands in the Q band region). ${ }^{b}$ This refers to the ratio between the Soret band and the most intense feature in the higher energy region (e.g. ratio between bands 11 and 17). ${ }^{c}$ The first and second numbers are taken from refs. 1 and 2, respectively. ${ }^{d}$ Ref. 2.

B. Elemental Analysis

\begin{tabular}{|l|l|l|l|l|l|l|l|l|l|l|l|l|}
\hline \multicolumn{3}{|c|}{$\left[\mathrm{Mn}{ }^{\mathrm{III}}(\mathrm{TPP}) \mathrm{F}\right]$} & \multicolumn{3}{c|}{$\left[\mathrm{Mn}^{\mathrm{III}}(\mathrm{TPP}) \mathrm{Cl}\right]$} & \multicolumn{3}{c|}{$\left[\mathrm{Mn}^{\mathrm{III}}(\mathrm{TPP}) \mathrm{Br}\right]$} & \multicolumn{3}{c|}{$\left[\mathrm{Mn}^{\mathrm{III}}(\mathrm{TPP})\right]^{a}$} \\
\hline Element & Theory & Expt. & Element & Theory & Expt. & Element & Theory & Expt. & Element & Theory & Expt. \\
\hline $\mathrm{C}$ & 76.96 & 75.09 & $\mathrm{C}$ & 75.16 & 74.84 & $\mathrm{C}$ & 70.69 & 69.01 & $\mathrm{C}$ & 66.51 & 63.12 \\
\hline $\mathrm{H}$ & 4.11 & 4.40 & $\mathrm{H}$ & 4.01 & 4.55 & $\mathrm{H}$ & 3.78 & 4.49 & $\mathrm{H}$ & 3.55 & 3.73 \\
\hline $\mathrm{N}$ & 8.16 & 8.08 & $\mathrm{~N}$ & 7.97 & 7.29 & $\mathrm{~N}$ & 7.50 & 7.62 & $\mathrm{~N}$ & 7.05 & 6.51 \\
\hline F & 2.77 & 2.72 & $\mathrm{Cl}$ & 5.04 & 5.33 & $\mathrm{Br}$ & 10.69 & 9.45 & $\mathrm{I}$ & 15.97 & 12.22 \\
\hline
\end{tabular}

${ }^{a}$ Based on several tests in our lab, [Mn $\left.{ }^{\mathrm{III}}(\mathrm{TPP}) \mathrm{I}\right]$ is observed to be extremely photolabile (Figure S11). The rather large deviation in the elemental analysis may arise from the degradation of the sample upon exposure to light during the elemental analysis procedure.

C. ${ }^{1} \mathrm{H}$ NMR signals for $\left[\mathrm{Mn}^{\mathrm{III}}(\mathrm{TPP}) \mathrm{X}\right]$ in $\mathrm{CD}_{2} \mathrm{Cl}_{2}$ in $\mathrm{ppm}$

\begin{tabular}{|c|c|c|c|c|}
\hline \multirow{2}{*}{$\mathrm{X}$} & \multicolumn{3}{|c|}{ phenyl $^{a}$} & \multirow{2}{*}{$\beta$-pyrrole ${ }^{a}$} \\
\hline & ortho & meta & para & \\
\hline $\mathrm{F}^{-}$ & $b$ & $8.37(8.15)$ & $7.39(7.3)$ & $-19.08(-19.7)$ \\
\hline $\mathrm{Cl}^{-}$ & $b$ & $8.46(8.3)$ & $7.45(7.3)$ & $-22.16(-22.3)$ \\
\hline $\mathrm{Br}$ & $b$ & $8.57(8.45)$ & $7.62(7.5)$ & $-23.56(-23.5)$ \\
\hline $\mathrm{I}^{-}$ & $b$ & $8.66(8.55)$ & $7.83(7.75)$ & $-26.04(-25.9)$ \\
\hline
\end{tabular}

${ }^{a}$ The values in parenthesis are taken from ref. 2 for comparison. ${ }^{b}$ The ortho proton peaks are broad and masked by the meta and para proton signals. This is consistent with ref. 2 . 
Table S1. Crystal structures of different $\left[\mathrm{Mn}^{\mathrm{III}}(\mathrm{TPP}) \mathrm{X}\right](\mathrm{X}=$ halide $)$ complexes.

\begin{tabular}{|l|l|l|l|}
\hline Ligand $(\mathrm{X})$ & $d(\mathrm{Mn}-\mathrm{X}), \AA$ & $d(\mathrm{Mn}-\mathrm{N}($ Porph) $), \AA$ & Mn-Ct (out-of-plane), ${ }^{a} \AA$ \\
\hline $\mathrm{Cl}^{b}$ & 2.389 & 2.019 & 0.258 \\
\hline $\mathrm{Cl}^{c}$ & 2.345 & $2.015(17)$ & 0.32 \\
\hline $\mathrm{Br}^{d}$ & 2.490 & $2.008(17)$ & 0.27 \\
\hline $\mathrm{I} \mathrm{(a)}{ }^{d}$ & 2.767 & $2.004(5)$ & 0.24 \\
\hline $\mathrm{I} \mathrm{(b)}{ }^{d}$ & 2.730 & $2.018(13)$ & 0.25 \\
\hline
\end{tabular}

${ }^{a}$ This parameter refers to the displacement of the Mn atom from the basal nitrogen plane. ${ }^{b}$ Ref.3. $n$-hexane was slowly diffused into a chloroform solution of $\left[\mathrm{Mn}^{\mathrm{III}}(\mathrm{TPP}) \mathrm{Cl}\right] .{ }^{c}$ Ref. 4 . Toluene was used as a crystallizing solvent. ${ }^{d}$ Ref. 5 . Toluene was used as a crystallizing solvent.

Table S2. Cartesian coordinates $[\AA]$ of $\left[\mathrm{Mn}^{\mathrm{III}}(\mathrm{TPP}) \mathrm{F}\right]$ obtained with B3LYP/LanL2DZ* (full geometry optimization). The structure is shown in Figure S8.

$\begin{array}{lrrr}\mathrm{C} & 4.22080100 & 0.68707100 & -0.27546000 \\ \mathrm{C} & 4.22174800 & -0.68056000 & -0.27745600 \\ \mathrm{C} & 2.84514100 & 1.10695100 & -0.15076900 \\ \mathrm{H} & 5.06794700 & 1.35676500 & -0.36672300 \\ \mathrm{C} & 2.84669500 & -1.10270600 & -0.15369800 \\ \mathrm{~N} & 2.01569700 & 0.00147500 & -0.09766800 \\ \mathrm{C} & 2.44731100 & 2.45042000 & -0.10160800 \\ \mathrm{H} & 5.06982200 & -1.34881400 & -0.37063500 \\ \mathrm{C} & 1.10330900 & 2.84606500 & -0.06717700 \\ \mathrm{C} & 2.45081100 & -2.44682100 & -0.10676600 \\ \mathrm{C} & 3.50950200 & 3.50854400 & -0.08071100 \\ \mathrm{C} & 0.68049100 & 4.22311100 & 0.02066500 \\ \mathrm{~N} & -0.00139800 & 2.01213100 & -0.11582900 \\ \mathrm{C} & 1.10744300 & -2.84443100 & -0.07012200 \\ \mathrm{C} & 3.51441600 & -3.50359900 & -0.08973100 \\ \mathrm{C} & 3.72251500 & 4.34167200 & -1.19620600 \\ \mathrm{C} & 4.31522200 & 3.68507900 & 1.06120200 \\ \mathrm{C} & -0.68688000 & 4.22211900 & 0.01863100 \\ \mathrm{C} & -1.10744800 & 2.84443000 & -0.07005300 \\ \mathrm{C} & 0.68687800 & -4.22211900 & 0.01859800 \\ \mathrm{~N} & 0.00139100 & -2.01213100 & -0.11583300 \\ \mathrm{H} & 1.34818700 & 5.07380100 & 0.08978000 \\ \mathrm{C} & 3.72629100 & -4.33429800 & -1.20724900 \\ \mathrm{C} & 4.32247300 & -3.68146600 & 1.05032300 \\ \mathrm{C} & 4.72138800 & 5.32701100 & -1.17312600 \\ \mathrm{C} & 5.30881400 & 4.67537300 & 1.08884400 \\ \mathrm{H} & 3.10963600 & 4.20859400 & -2.08834000 \\ \mathrm{H} & 4.15151300 & 3.05102000 & 1.93301800 \\ \mathrm{C} & -2.45081700 & 2.44682000 & -0.10662700 \\ \mathrm{C} & -1.10331400 & -2.84606500 & -0.06712200 \\ \mathrm{C} & -0.68049200 & -4.22311200 & 0.02068100 \\ \mathrm{H} & -1.35600400 & 5.07184500 & 0.08578000 \\ \mathrm{H} & 1.35600600 & -5.07184400 & 0.08571900\end{array}$




$\begin{array}{lrrr}\mathrm{C} & 4.72637600 & -5.31849500 & -1.18800500 \\ \mathrm{C} & 5.31728600 & -4.67063000 & 1.07413700 \\ \mathrm{H} & 3.1152700 & -4.20023300 & -2.09793700 \\ \mathrm{H} & 4.15965700 & -3.04934900 & 1.92371700 \\ \mathrm{C} & 5.51715800 & 5.49881400 & -0.02921300 \\ \mathrm{H} & 4.87771500 & 5.95839200 & -2.04840300 \\ \mathrm{H} & 5.91777500 & 4.80371200 & 1.98423400 \\ \mathrm{C} & -2.84670400 & 1.10270500 & -0.15354500 \\ \mathrm{C} & -2.44731700 & -2.45042100 & -0.10148100 \\ \mathrm{C} & -3.51442100 & 3.50359900 & -0.08953300 \\ \mathrm{H} & -1.34818400 & -5.07380300 & 0.08982200 \\ \mathrm{C} & 5.52452000 & -5.49160100 & -0.04594400 \\ \mathrm{H} & 4.88178700 & -5.94796900 & -2.06481600 \\ \mathrm{H} & 5.92807700 & -4.80001800 & 1.96813000 \\ \mathrm{H} & 6.29124400 & 6.26661800 & -0.00918800 \\ \mathrm{C} & -4.22176200 & 0.68056000 & -0.27724100 \\ \mathrm{C} & -2.84515000 & -1.10695100 & -0.15062200 \\ \mathrm{C} & -3.50950700 & -3.50854400 & -0.08052600 \\ \mathrm{~N} & -2.01570200 & -0.00147600 & -0.09756200 \\ \mathrm{C} & -4.32241800 & 3.68146000 & 1.05056400 \\ \mathrm{C} & -3.72635100 & 4.33430700 & -1.20703500 \\ \mathrm{H} & 6.29957700 & -6.25849300 & -0.02890600 \\ \mathrm{C} & -4.22081600 & -0.68707100 & -0.27523600 \\ \mathrm{H} & -5.06984100 & 1.34881400 & -0.37038000 \\ \mathrm{C} & -3.72257600 & -4.34167800 & -1.19600600 \\ \mathrm{C} & -4.31516800 & -3.68507400 & 1.06142900 \\ \mathrm{C} & -5.31722900 & 4.67062600 & 1.07443500 \\ \mathrm{C} & -4.72643400 & 5.31850500 & -1.18773300 \\ \mathrm{H} & -4.15955800 & 3.04933900 & 1.92394600 \\ \mathrm{H} & -3.11163300 & 4.20024600 & -2.09775400 \\ \mathrm{H} & -5.06796800 & -1.35676600 & -0.36645400 \\ \mathrm{C} & -4.72144800 & -5.32701700 & -1.17287100 \\ \mathrm{C} & -5.30876000 & -4.67536700 & 1.08912600 \\ \mathrm{C} & -5.52452000 & 5.49160300 & -0.04563100 \\ \mathrm{H} & -3.10974100 & -4.20860400 & -2.08817100 \\ \mathrm{H} & -4.15141500 & -3.05101000 & 1.93323300 \\ \mathrm{H} & -5.92797300 & 4.80000900 & 1.96846000 \\ \mathrm{H} & -4.88188900 & 5.94798400 & -2.06453200 \\ \mathrm{C} & -5.51716100 & -5.49881500 & -0.02891600 \\ \mathrm{H} & -4.87781800 & -5.95840300 & -2.04813600 \\ \mathrm{H} & -5.91767500 & -4.80370200 & 1.98454800 \\ \mathrm{H} & -6.29957500 & 6.25849700 & -0.02854800 \\ \mathrm{H} & -6.29124400 & -6.26661800 & -0.00884800 \\ & 0.00000600 & 0.00000000 & 0.19566900 \\ \mathrm{H} & 0.00006700 & -0.00000300 & 2.56028900\end{array}$


Table S3. Cartesian coordinates $[\AA]$ of $\left[\mathrm{Mn}^{\mathrm{III}}(\mathrm{TPP}) \mathrm{Cl}\right]$ obtained with B3LYP/LanL2DZ* (full geometry optimization). The structure is shown in Figure 1.

\begin{tabular}{|c|c|c|c|}
\hline C & 4.22079800 & 0.68707900 & -0.33204100 \\
\hline $\mathrm{C}$ & 4.22174800 & -0.68055200 & -0.33403700 \\
\hline $\mathrm{C}$ & 2.84513700 & 1.10695700 & -0.20735000 \\
\hline $\mathrm{H}$ & 5.06794300 & 1.35677600 & -0.42330400 \\
\hline $\mathrm{C}$ & 2.84669600 & -1.10270000 & -0.21027900 \\
\hline $\mathrm{N}$ & 2.01569600 & 0.00148000 & -0.15424900 \\
\hline $\mathrm{C}$ & 2.44730500 & 2.45042500 & -0.15818900 \\
\hline $\mathrm{H}$ & 5.06982400 & -1.34880400 & -0.42721600 \\
\hline $\mathrm{C}$ & 1.10330200 & 2.84606700 & -0.12375800 \\
\hline $\mathrm{C}$ & 2.45081400 & -2.44681600 & -0.16334700 \\
\hline $\mathrm{C}$ & 3.50949300 & 3.50855100 & -0.13729200 \\
\hline $\mathrm{C}$ & 0.68048200 & 4.22311200 & -0.03591600 \\
\hline $\mathrm{N}$ & -0.00140400 & 2.01213100 & -0.17241000 \\
\hline $\mathrm{C}$ & 1.10744700 & -2.84442900 & -0.12670300 \\
\hline $\mathrm{C}$ & 3.51442200 & -3.50359200 & -0.14631200 \\
\hline $\mathrm{C}$ & 3.72250500 & 4.34167900 & -1.25278700 \\
\hline $\mathrm{C}$ & 4.31521300 & 3.68508800 & 1.00462100 \\
\hline $\mathrm{C}$ & -0.68689000 & 4.22211800 & -0.03795000 \\
\hline $\mathrm{C}$ & -1.10745500 & 2.84442800 & -0.12663400 \\
\hline $\mathrm{C}$ & 0.68688600 & -4.22211800 & -0.03798300 \\
\hline $\mathrm{N}$ & 0.00139400 & -2.01213100 & -0.17241400 \\
\hline $\mathrm{H}$ & 1.34817500 & 5.07380300 & 0.03319900 \\
\hline $\mathrm{C}$ & 3.72629800 & -4.33429100 & -1.26383000 \\
\hline $\mathrm{C}$ & 4.32247900 & -3.68145700 & 0.99374200 \\
\hline $\mathrm{C}$ & 4.72137600 & 5.32702100 & -1.22970700 \\
\hline $\mathrm{C}$ & 5.30880300 & 4.67538300 & 1.03226300 \\
\hline $\mathrm{H}$ & 3.10962600 & 4.20860000 & -2.14492100 \\
\hline $\mathrm{H}$ & 4.15150600 & 3.05102800 & 1.87643700 \\
\hline $\mathrm{C}$ & -2.45082300 & 2.44681600 & -0.16320800 \\
\hline $\mathrm{C}$ & -1.10331000 & -2.84606700 & -0.12370300 \\
\hline $\mathrm{C}$ & -0.68048500 & -4.22311300 & -0.03590000 \\
\hline $\mathrm{H}$ & -1.35601600 & 5.07184200 & 0.02919900 \\
\hline $\mathrm{H}$ & 1.35601500 & -5.07184200 & 0.02913800 \\
\hline $\mathrm{C}$ & 4.72638500 & -5.31848500 & -1.24458600 \\
\hline $\mathrm{C}$ & 5.31729400 & -4.67062000 & 1.01755600 \\
\hline $\mathrm{H}$ & 3.11153400 & -4.20022600 & -2.15451800 \\
\hline $\mathrm{H}$ & 4.15966200 & -3.04934100 & 1.86713600 \\
\hline $\mathrm{C}$ & 5.51714600 & 5.49882600 & -0.08579400 \\
\hline $\mathrm{H}$ & 4.87770200 & 5.95840200 & -2.10498400 \\
\hline $\mathrm{H}$ & 5.91776400 & 4.80372400 & 1.92765300 \\
\hline $\mathrm{C}$ & -2.84670800 & 1.10269900 & -0.21012600 \\
\hline $\mathrm{C}$ & -2.44731400 & -2.45042500 & -0.15806200 \\
\hline $\mathrm{C}$ & -3.51443000 & 3.50359200 & -0.14611400 \\
\hline $\mathrm{H}$ & -1.34817500 & -5.07380500 & 0.03324100 \\
\hline $\mathrm{C}$ & 5.52452900 & -5.49158900 & -0.10252500 \\
\hline $\mathrm{H}$ & 4.88179700 & -5.94795900 & -2.12139700 \\
\hline $\mathrm{H}$ & 5.92808500 & -4.80000600 & 1.91154900 \\
\hline $\mathrm{H}$ & 6.29123000 & 6.26663000 & -0.06576900 \\
\hline
\end{tabular}




$\begin{array}{lrrr}\mathrm{C} & -4.22176500 & 0.68055200 & -0.33382200 \\ \mathrm{C} & -2.84514900 & -1.10695700 & -0.20720300 \\ \mathrm{C} & -3.50950100 & -3.50855100 & -0.13710700 \\ \mathrm{~N} & -2.01570400 & -0.00148000 & -0.15414300 \\ \mathrm{C} & -4.32242700 & 3.68145200 & 0.99398300 \\ \mathrm{C} & -3.72636100 & 4.33430000 & -1.26361600 \\ \mathrm{H} & 6.29958800 & -6.25848000 & -0.08548700 \\ \mathrm{C} & -4.22081600 & -0.68707900 & -0.33181700 \\ \mathrm{H} & -5.06984500 & 1.34880400 & -0.42696100 \\ \mathrm{C} & -3.72256900 & -4.34168500 & -1.25258700 \\ \mathrm{C} & -4.31516200 & -3.68508300 & 1.00484800 \\ \mathrm{C} & -5.31724000 & 4.67061600 & 1.01785400 \\ \mathrm{C} & -4.72644600 & 5.31849500 & -1.24431400 \\ \mathrm{H} & -4.15956600 & 3.04933100 & 1.86736500 \\ \mathrm{H} & -3.11164300 & 4.20023900 & -2.15433500 \\ \mathrm{H} & -5.06796600 & -1.35677600 & -0.42303500 \\ \mathrm{C} & -4.72143900 & -5.32702700 & -1.22945200 \\ \mathrm{C} & -5.30875200 & -4.67537700 & 1.03254500 \\ \mathrm{C} & -5.52453200 & 5.49159200 & -0.10221200 \\ \mathrm{H} & -3.10973400 & -4.20861100 & -2.14475200 \\ \mathrm{H} & -4.15141100 & -3.05101800 & 1.87665200 \\ \mathrm{H} & -5.92798400 & 4.79999700 & 1.91187900 \\ \mathrm{H} & -4.88190200 & 5.94797400 & -2.12111300 \\ \mathrm{C} & -5.51715100 & -5.49882600 & -0.08549700 \\ \mathrm{H} & -4.87780800 & -5.95841300 & -2.10471700 \\ \mathrm{H} & -5.91766700 & -4.80371400 & 1.92796700 \\ \mathrm{H} & -6.29958900 & 6.25848400 & -0.08512900 \\ \mathrm{H} & -6.29123300 & -6.26663000 & -0.06542900 \\ \mathrm{Mn} & 0.00000400 & 0.00000000 & 0.13908800 \\ \mathrm{Cl} & 0.00006500 & -0.00000300 & 2.50370800\end{array}$

Table S4. Cartesian coordinates $[\AA]$ of $\left[\mathrm{Mn}^{\mathrm{III}}(\mathrm{TPP}) \mathrm{Br}\right]$ obtained with B3LYP/LanL2DZ* (full geometry optimization). The structure is shown in Figure S8.

$\begin{array}{lrrr}\mathrm{Mn} & -0.00006100 & 0.00000000 & -0.00555000 \\ \mathrm{~N} & -0.00012600 & 2.01135400 & -0.30418100 \\ \mathrm{~N} & 2.01415700 & -0.00000200 & -0.28619600 \\ \mathrm{~N} & -2.01430300 & 0.00000200 & -0.28608500 \\ \mathrm{C} & -1.10556800 & 2.84520400 & -0.25997900 \\ \mathrm{C} & -0.68382100 & 4.22250900 & -0.17551600 \\ \mathrm{C} & 0.68361100 & 4.22249600 & -0.17551000 \\ \mathrm{C} & 1.10534800 & 2.84518200 & -0.25996400 \\ \mathrm{C} & 2.44887300 & 2.44873800 & -0.29401500 \\ \mathrm{C} & 2.84529600 & 1.10500600 & -0.33893200 \\ \mathrm{C} & 4.22070300 & 0.68382400 & -0.45824400 \\ \mathrm{C} & -4.22090800 & 0.68383400 & -0.45760200 \\ \mathrm{C} & -2.84547900 & 1.10501200 & -0.33864300 \\ \mathrm{C} & -2.44909000 & 2.44875500 & -0.29389700 \\ \mathrm{C} & -3.51196500 & 3.50632000 & -0.27591400 \\ \mathrm{C} & -4.31339300 & 3.68996400 & 0.86786200\end{array}$




\begin{tabular}{|c|c|c|c|}
\hline $\mathrm{C}$ & -5.30738400 & 4.67996300 & 0.89251900 \\
\hline $\mathrm{C}$ & -5.52024100 & 5.49586900 & -0.23021000 \\
\hline $\mathrm{C}$ & -4.72860400 & 5.31692700 & -1.37590200 \\
\hline $\mathrm{C}$ & -3.72936900 & 4.33187300 & -1.39613500 \\
\hline $\mathrm{C}$ & 3.51176000 & 3.50630200 & -0.27605400 \\
\hline $\mathrm{C}$ & 4.31351000 & 3.68960800 & 0.86755700 \\
\hline $\mathrm{C}$ & 5.30752700 & 4.67957500 & 0.89220700 \\
\hline $\mathrm{C}$ & 5.52010700 & 5.49576800 & -0.23037300 \\
\hline $\mathrm{C}$ & 4.72816100 & 5.31715400 & -1.37589400 \\
\hline $\mathrm{C}$ & 3.72887700 & 4.33214000 & -1.39611400 \\
\hline $\mathrm{Br}$ & 0.00093100 & 0.00000000 & 2.54264100 \\
\hline $\mathrm{H}$ & -1.35237200 & 5.07277900 & -0.10982400 \\
\hline $\mathrm{H}$ & 1.35215400 & 5.07276700 & -0.10977400 \\
\hline $\mathrm{H}$ & 5.06857900 & 1.35286400 & -0.54717900 \\
\hline $\mathrm{H}$ & -5.06880200 & 1.35288300 & -0.54630700 \\
\hline $\mathrm{H}$ & -4.14612400 & 3.06182100 & 1.74326100 \\
\hline $\mathrm{H}$ & -5.91307300 & 4.81393000 & 1.78928100 \\
\hline $\mathrm{H}$ & -6.29461200 & 6.26342000 & -0.21241500 \\
\hline $\mathrm{H}$ & -4.88838100 & 5.94250300 & -2.25470100 \\
\hline $\mathrm{H}$ & -3.11956400 & 4.19329700 & -2.28955000 \\
\hline $\mathrm{H}$ & 4.14645000 & 3.06123000 & 1.74282500 \\
\hline $\mathrm{H}$ & 5.91345700 & 4.81331000 & 1.78884100 \\
\hline $\mathrm{H}$ & 6.29451900 & 6.26327800 & -0.21258200 \\
\hline $\mathrm{H}$ & 4.88772700 & 5.94294600 & -2.25457600 \\
\hline $\mathrm{H}$ & 3.11882200 & 4.19383500 & -2.28939900 \\
\hline $\mathrm{N}$ & -0.00013100 & -2.01135400 & -0.30418100 \\
\hline $\mathrm{C}$ & -1.10557400 & -2.84520200 & -0.25997800 \\
\hline $\mathrm{C}$ & -0.68383000 & -4.22250800 & -0.17551600 \\
\hline $\mathrm{C}$ & 0.68360100 & -4.22249800 & -0.17551000 \\
\hline $\mathrm{C}$ & 1.10534200 & -2.84518500 & -0.25996400 \\
\hline $\mathrm{C}$ & 2.44886700 & -2.44874400 & -0.29401600 \\
\hline $\mathrm{C}$ & 2.84529300 & -1.10501200 & -0.33893200 \\
\hline $\mathrm{C}$ & 4.22070200 & -0.68383300 & -0.45824400 \\
\hline $\mathrm{H}$ & 5.06857600 & -1.35287500 & -0.54718000 \\
\hline $\mathrm{C}$ & 3.51175200 & -3.50631000 & -0.27605500 \\
\hline $\mathrm{C}$ & 4.31350200 & -3.68961700 & 0.86755500 \\
\hline $\mathrm{C}$ & 5.30751800 & -4.67958600 & 0.89220400 \\
\hline $\mathrm{C}$ & 5.52009400 & -5.49578000 & -0.23037500 \\
\hline $\mathrm{C}$ & 4.72814700 & -5.31716500 & -1.37589600 \\
\hline $\mathrm{C}$ & 3.72886600 & -4.33214900 & -1.39611500 \\
\hline $\mathrm{H}$ & 3.11881000 & -4.19384300 & -2.28940000 \\
\hline $\mathrm{H}$ & 4.88771200 & -5.94295900 & -2.25457700 \\
\hline $\mathrm{H}$ & 6.29450500 & -6.26329200 & -0.21258400 \\
\hline $\mathrm{H}$ & 5.91344800 & -4.81332200 & 1.78883800 \\
\hline $\mathrm{H}$ & 4.14644500 & -3.06123900 & 1.74282300 \\
\hline $\mathrm{H}$ & 1.35214300 & -5.07277000 & -0.10977400 \\
\hline $\mathrm{H}$ & -1.35238400 & -5.07277600 & -0.10982300 \\
\hline $\mathrm{C}$ & -2.44909500 & -2.44874900 & -0.29389600 \\
\hline $\mathrm{C}$ & -2.84548100 & -1.10500600 & -0.33864200 \\
\hline $\mathrm{C}$ & -4.22091000 & -0.68382500 & -0.45760200 \\
\hline $\mathrm{H}$ & -5.06880500 & -1.35287200 & -0.54630600 \\
\hline
\end{tabular}




$\begin{array}{lrrr}\mathrm{C} & -3.51197300 & -3.50631200 & -0.27591300 \\ \mathrm{C} & -4.31340200 & -3.68995300 & 0.86786400 \\ \mathrm{C} & -5.30739500 & -4.67995000 & 0.89252100 \\ \mathrm{C} & -5.52025400 & -5.49585700 & -0.23020800 \\ \mathrm{C} & -4.72861600 & -5.31691700 & -1.37589900 \\ \mathrm{C} & -3.72937800 & -4.33186500 & -1.39613300 \\ \mathrm{H} & -3.11957300 & -4.19329200 & -2.28954800 \\ \mathrm{H} & -4.88839400 & -5.94249300 & -2.25469800 \\ \mathrm{H} & -6.29462700 & -6.26340500 & -0.21241200 \\ \mathrm{H} & -5.91308500 & -4.81391500 & 1.78928200 \\ \mathrm{H} & -4.14613100 & -3.06181000 & 1.74326200\end{array}$

Table S5. Cartesian coordinates $[\AA]$ of $\left[\mathrm{Mn}^{\mathrm{III}}(\mathrm{TPP}) \mathrm{I}\right]$ obtained with B3LYP/LanL2DZ* (full geometry optimization). The structure is shown in Figure S8.

$\begin{array}{lrrr}\mathrm{Mn} & 0.00085000 & -0.00010500 & -0.17005200 \\ \mathrm{~N} & 0.01350400 & 2.00837300 & -0.43467700 \\ \mathrm{~N} & 2.01325400 & -0.01492400 & -0.43607600 \\ \mathrm{~N} & -0.01086700 & -2.00805200 & -0.43522700 \\ \mathrm{~N} & -2.01098400 & 0.01597400 & -0.43989300 \\ \mathrm{C} & -1.08126100 & 2.84893100 & -0.30055800 \\ \mathrm{C} & -0.64122900 & 4.21575000 & -0.16763700 \\ \mathrm{C} & 0.72394800 & 4.20675500 & -0.25184000 \\ \mathrm{C} & 1.12791600 & 2.83250600 & -0.42009900 \\ \mathrm{C} & 2.46484300 & 2.42770500 & -0.53898100 \\ \mathrm{C} & 2.84907100 & 1.07978100 & -0.57702300 \\ \mathrm{C} & 4.21418800 & 0.64052500 & -0.73774000 \\ \mathrm{C} & 4.20676000 & -0.72503300 & -0.65499800 \\ \mathrm{C} & 2.83536100 & -1.12797000 & -0.45893800 \\ \mathrm{C} & 2.43109200 & -2.46492500 & -0.33088000 \\ \mathrm{C} & 1.08359900 & -2.84870300 & -0.30093600 \\ \mathrm{C} & 0.64326100 & -4.21551600 & -0.16843900 \\ \mathrm{C} & -0.72182000 & -4.20637000 & -0.25440800 \\ \mathrm{C} & -1.12557400 & -2.83183200 & -0.42105500 \\ \mathrm{C} & -2.46220100 & -2.42676800 & -0.54152000 \\ \mathrm{C} & -2.84649700 & -1.07891900 & -0.58102700 \\ \mathrm{C} & -4.21177100 & -0.63991700 & -0.74137200 \\ \mathrm{C} & -4.20442300 & 0.72572700 & -0.65986800 \\ \mathrm{C} & -2.83320300 & 1.12878000 & -0.46253600 \\ \mathrm{C} & -2.42876900 & 2.46547500 & -0.33234900 \\ \mathrm{C} & -3.48627000 & 3.52251000 & -0.23673900 \\ \mathrm{C} & -4.32523600 & 3.58342600 & 0.89363000 \\ \mathrm{C} & -5.31955500 & 4.56796300 & 0.99360100 \\ \mathrm{C} & -5.49682300 & 5.50099200 & -0.04051000 \\ \mathrm{C} & -4.66967300 & 5.44384400 & -1.17364700 \\ \mathrm{C} & -3.66970800 & 4.46420500 & -1.26848700 \\ \mathrm{C} & 3.52919200 & 3.48067700 & -0.60260000 \\ \mathrm{C} & 3.62085100 & 4.34134600 & -1.71428000 \\ \mathrm{C} & 4.61752800 & 5.32678300 & -1.77702600 \\ \mathrm{C} & 5.53313000 & 5.47257900 & -0.72266600\end{array}$




$\begin{array}{cccc}\mathrm{C} & 5.44566700 & 4.62333400 & 0.39210600 \\ \mathrm{C} & 4.45413800 & 3.63243800 & 0.44939700 \\ \mathrm{C} & 3.48858900 & -3.52193400 & -0.23561600 \\ \mathrm{C} & 4.32547900 & -3.58544800 & 0.89611400 \\ \mathrm{C} & 5.31932500 & -4.57051200 & 0.99588200 \\ \mathrm{C} & 5.49797000 & -5.50169200 & -0.03962300 \\ \mathrm{C} & 4.67253300 & -5.44229000 & -1.17392400 \\ \mathrm{C} & 3.67297300 & -4.46224800 & -1.26851200 \\ \mathrm{C} & -3.52680100 & -3.47977200 & -0.60490900 \\ \mathrm{C} & -3.62373900 & -4.33497600 & -1.72030800 \\ \mathrm{C} & -4.62114700 & -5.31968700 & -1.78322700 \\ \mathrm{C} & -5.53189500 & -5.47016700 & -0.72531500 \\ \mathrm{C} & -5.43921500 & -4.62617200 & 0.39299200 \\ \mathrm{C} & -4.44678700 & -3.63612100 & 0.45064600 \\ \mathrm{I} & -0.00643900 & -0.00200300 & 2.62660800 \\ \mathrm{H} & -1.29498900 & 5.06680800 & -0.01814200 \\ \mathrm{H} & 1.40259700 & 5.04873900 & -0.18350900 \\ \mathrm{H} & 5.06169600 & 1.29509800 & -0.90354100 \\ \mathrm{H} & 5.04650900 & -1.40457400 & -0.74133600 \\ \mathrm{H} & 1.29686500 & -5.06669200 & -0.01891300 \\ \mathrm{H} & -1.40061700 & -5.04837400 & -0.18771800 \\ \mathrm{H} & -5.05918300 & -1.29479300 & -0.90650700 \\ \mathrm{H} & -5.04416000 & 1.40524000 & -0.74652300 \\ \mathrm{H} & -4.18506600 & 2.86473000 & 1.70139600 \\ \mathrm{H} & -5.95314000 & 4.60639800 & 1.88019800 \\ \mathrm{H} & -6.27179700 & 6.26438600 & 0.03554300 \\ \mathrm{H} & -4.80311200 & 6.15973100 & -1.98535300 \\ \mathrm{H} & -3.03525100 & 4.41850800 & -2.15423700 \\ \mathrm{H} & 2.91426900 & 4.22918200 & -2.53723600 \\ \mathrm{H} & 4.67861300 & 5.97863100 & -2.64909500 \\ \mathrm{H} & 6.30585500 & 6.24061500 & -0.76862600 \\ \mathrm{H} & 6.14698300 & 4.73263100 & 1.21986500 \\ \mathrm{H} & 4.38331200 & 2.98135600 & 1.32105900 \\ \mathrm{H} & 4.18407600 & -2.86840600 & 1.70514000 \\ \mathrm{H} & 5.95135500 & -4.61078600 & 1.88350900 \\ \mathrm{H} & -2.27257100 & -6.26548100 & 0.03622700 \\ \mathrm{H} & -6.30505300 & -6.23777500 & -0.77131400 \\ & -6.13691400 & -4.73909900 & 1.22331200 \\ \mathrm{H} & -4.37186000 & -2.98899300 & 1.32490400\end{array}$


Table S6. TD-DFT (B3LYP/LanL2DZ*) results for [ $\left.\mathrm{Mn}{ }^{\mathrm{III}}(\mathrm{TPP}) \mathrm{Cl}\right]$ (Figure 1). Contributions are given in \%.

\begin{tabular}{|c|c|c|c|c|c|c|}
\hline TD-DFT No. & 8 & 9 & 11 & 10 & 15 & 17 \\
\hline $\begin{array}{l}\text { TD-DFT Excitation energy, } \\
\mathrm{cm}^{-1} \\
(\mathrm{~nm}, \mathrm{f})\end{array}$ & $\begin{array}{l}16614(602, \\
0.0043)\end{array}$ & $\begin{array}{l}16622(602, \\
0.0093)\end{array}$ & $\begin{array}{l}17058(586, \\
0.0139)\end{array}$ & $\begin{array}{l}17057(586, \\
0.0218)\end{array}$ & $\begin{array}{l}20798(481 \\
0.0003)\end{array}$ & $\begin{array}{l}21244(471 \\
0.0205)\end{array}$ \\
\hline $\begin{array}{l}\text { Experimental energy, } \mathrm{cm}^{-1} \\
(\mathrm{~nm}, \mathrm{f})\end{array}$ & $\begin{array}{l}15581, \text { band } 2 \\
(630,0.0072)\end{array}$ & $\begin{array}{l}16282, \text { band } 3 \\
(614,0.0288) \\
\end{array}$ & $\begin{array}{l}16879, \text { band } 4 \\
(592,0.0118)\end{array}$ & $\begin{array}{l}17273, \text { band } 5 \\
(579,0.0328)\end{array}$ & $\begin{array}{l}18290, \text { band } 6 \\
(547,0.0181)\end{array}$ & $\begin{array}{l}18890, \text { band } 7 \\
(529,0.0186)\end{array}$ \\
\hline $\begin{array}{c}\text { Experimental polarizations } \\
\% \mathrm{x} \\
\% \mathrm{y} \\
\% \mathrm{z} \\
\end{array}$ & $\begin{array}{c}4 \\
42[31-55] \\
53[40-63] \\
\end{array}$ & $\begin{array}{c}56[54-63] \\
2-3 \\
41[34-43] \\
\end{array}$ & $\begin{array}{c}63[56-71] \\
3-4 \\
33[25-41] \\
\end{array}$ & $\begin{array}{c}74-76 \\
19 \\
5-7 \\
\end{array}$ & $\begin{array}{c}80[82-84] \\
5 \\
13[11-15] \\
\end{array}$ & $\begin{array}{c}81-84 \\
9 \\
7-9 \\
\end{array}$ \\
\hline \multicolumn{7}{|c|}{ Inner Porphyrin Excitations ( $x y$-polarized) } \\
\hline$\pi \rightarrow \pi^{*(0)}$ & 12 & 32 & 71 & 87 & & 7 \\
\hline \multicolumn{7}{|l|}{$\pi \rightarrow \pi^{*(1)}$} \\
\hline \multicolumn{7}{|l|}{$\pi \rightarrow \pi^{*(2)}$} \\
\hline \multicolumn{7}{|c|}{ Porphyrin to Fe Excitations ( $x y$-polarized) } \\
\hline \multicolumn{7}{|l|}{$\mathrm{CT}^{(1)}$} \\
\hline \multicolumn{7}{|l|}{$\mathrm{CT}^{(2)}$} \\
\hline \multicolumn{7}{|l|}{$\mathrm{CT}^{(3)}$} \\
\hline \multicolumn{7}{|l|}{$\mathrm{CT}^{(4)}$} \\
\hline \multicolumn{7}{|l|}{$\mathrm{CT}^{(5)}$} \\
\hline \multicolumn{7}{|l|}{$\mathrm{CT}^{(6)}$} \\
\hline \multicolumn{7}{|l|}{$\mathrm{CT}^{(7)}$} \\
\hline \multicolumn{7}{|c|}{ Chlorine to Fe Excitations ( $z$-polarized) } \\
\hline $\mathrm{CT}^{(\mathrm{Cl}, \pi)}$ & & & & & 100 & \\
\hline \multicolumn{7}{|l|}{$\mathrm{CT}^{(\mathrm{Cl}, \sigma)}$} \\
\hline Others & $\begin{array}{l}\mathrm{Cl}\left(\mathrm{p}_{\mathrm{y}}\right) \mathrm{d}_{\mathrm{yz}} \rightarrow \\
\mathrm{d}_{\mathrm{x}}^{2} \mathrm{y}^{2}(\mathbf{6 8}), \\
\mathrm{d}_{\mathrm{yz}} \rightarrow \mathrm{d}_{\mathrm{x}-\mathrm{y}^{2}}^{2} \\
(15)\end{array}$ & $\begin{array}{l}\mathrm{Cl}\left(\mathrm{p}_{\mathrm{x}}\right)_{-} \mathrm{d}_{\mathrm{xz}} \rightarrow \\
\mathrm{d}_{\mathrm{x}^{2}-\mathrm{y}}^{2} \mathbf{( 5 3 )}\end{array}$ & $\begin{array}{l}\mathrm{Cl}\left(\mathrm{p}_{\mathrm{x}}\right)_{-} \mathrm{d}_{\mathrm{xz}} \rightarrow \\
\mathrm{d}_{\mathrm{x}-\mathrm{y}}^{2}(25)\end{array}$ & $\begin{array}{l}\mathrm{Cl}\left(\mathrm{p}_{\mathrm{y}}\right)_{-} \mathrm{d}_{\mathrm{yz}} \rightarrow \\
\mathrm{d}_{\mathrm{x}-\mathrm{y}}^{2}(8)\end{array}$ & & $\begin{array}{l}\mathrm{E}_{\mathrm{g}}(\pi) \rightarrow \mathrm{d}_{\mathrm{x}-\mathrm{y}}^{2}{ }^{2} \\
(\mathbf{5 7}), \\
\mathrm{Cl}\left(\mathrm{p}_{\mathrm{y}}\right) \mathrm{d}_{\mathrm{yz}} \rightarrow \\
\mathrm{d}_{\mathrm{x}}^{2}-\mathrm{y}^{2}(17)\end{array}$ \\
\hline $\begin{array}{c}\text { TD-DFT polarizations } \\
\% \mathrm{x} \\
\% \mathrm{y} \\
\% \mathrm{z} \\
\end{array}$ & $\begin{array}{c}2 \\
98 \\
0 \\
\end{array}$ & $\begin{array}{c}100 \\
0 \\
0\end{array}$ & $\begin{array}{c}96 \\
4 \\
0 \\
\end{array}$ & $\begin{array}{c}2 \\
98 \\
0\end{array}$ & $\begin{array}{c}0 \\
0 \\
100 \\
\end{array}$ & $\begin{array}{c}0 \\
100 \\
0 \\
\end{array}$ \\
\hline
\end{tabular}


Table S6. TD-DFT (B3LYP/LanL2DZ*) results on [ $\left.\mathrm{Mn}^{\mathrm{III}}(\mathrm{TPP}) \mathrm{Cl}\right]$ (Figure 1). Contributions are given in \%.

\begin{tabular}{|c|c|c|c|c|c|c|}
\hline TD-DFT No. & 19 & 20 & 21 & 25 & 45 & 46 \\
\hline $\begin{array}{l}\text { TD-DFT Excitation } \\
\text { energy, } \mathrm{cm}^{-1} \\
(\mathrm{~nm}, \mathrm{f})\end{array}$ & $\begin{array}{l}21573(464, \\
0.1425)\end{array}$ & $\begin{array}{l}21595(463 \\
0.1808)\end{array}$ & $\begin{array}{l}21977(455 \\
0.0093)\end{array}$ & $\begin{array}{l}22955(436, \\
0.0447)\end{array}$ & $\begin{array}{l}27809(360 \\
0.0554)\end{array}$ & $\begin{array}{l}27824(359 \\
0.0760)\end{array}$ \\
\hline $\begin{array}{l}\text { Experimental energy, } \mathrm{cm}^{-1} \\
(\mathrm{~nm}, \mathrm{f})\end{array}$ & $\begin{array}{l}20750, \text { band } 10 \\
(482,0.0849)\end{array}$ & $\begin{array}{l}21000, \text { band } 11 \\
(476,0.1332)\end{array}$ & $\begin{array}{l}21240, \text { band } 12 \\
(471,0.0887)\end{array}$ & $\begin{array}{c}22500, \text { band } \\
14(444, \\
0.0659)\end{array}$ & \multicolumn{2}{|c|}{23264 , band $15(430,0.0549)$} \\
\hline $\begin{array}{c}\text { Experimental polarizations } \\
\% \mathrm{x} \\
\% \mathrm{y} \\
\% \mathrm{z} \\
\end{array}$ & $\begin{array}{c}79[65-87] \\
19[11-33] \\
2 \\
\end{array}$ & $\begin{array}{c}98 \\
2\end{array}$ & $\begin{array}{c}\text { Too weak to } \\
\text { generate VTVH } \\
\text { data } \\
\end{array}$ & $\begin{array}{c}29[25-34] \\
65[60-69] \\
5-6 \\
\end{array}$ & \multicolumn{2}{|c|}{$\begin{array}{c}96-98 \\
2-4\end{array}$} \\
\hline \multicolumn{7}{|c|}{ Inner Porphyrin Excitations ( $x y$-polarized) } \\
\hline$\pi \rightarrow \pi^{*(0)}$ & 42 & 49 & & 4 & & \\
\hline$\pi \rightarrow \pi^{*(1)}$ & & & & 6 & 43 & \\
\hline$\pi \rightarrow \pi^{*(2)}$ & & & & 4 & & \\
\hline \multicolumn{7}{|c|}{ Porphyrin to Fe Excitations ( $x y$-polarized) } \\
\hline $\mathrm{CT}^{(1)}$ & 3 & 3 & & & & 3 \\
\hline $\mathrm{CT}^{(2)}$ & 5 & 8 & & 45 & & \\
\hline \multicolumn{7}{|l|}{$\mathrm{CT}^{(3)}$} \\
\hline $\mathrm{CT}^{(4)}$ & & & & & 6 & \\
\hline \multicolumn{7}{|l|}{$\mathrm{CT}^{(5)}$} \\
\hline \multicolumn{7}{|l|}{$\mathrm{CT}^{(6)}$} \\
\hline $\mathrm{CT}^{(7)}$ & & & 1 & & & \\
\hline \multicolumn{7}{|c|}{ Chlorine to Fe Excitations ( $z$-polarized) } \\
\hline $\mathrm{CT}^{(\mathrm{Cl}, \pi)}$ & & & 95 & & 4 & \\
\hline $\mathrm{CT}^{(\mathrm{Cl}, \sigma)}$ & & & 1 & & & \\
\hline Others & $\begin{array}{lr}\mathrm{Cl}\left(\mathrm{p}_{\mathrm{z}}\right)_{-} \mathrm{d}_{z^{2}} & + \\
\mathrm{A}_{2 \mathrm{u}} \rightarrow \mathrm{E}_{\mathrm{g}} & \text { (37) }\end{array}$ & $\begin{array}{l}\mathrm{Cl}\left(\mathrm{p}_{\mathrm{z}}\right) \mathrm{d}_{z^{2}}+\mathrm{A}_{2 \mathrm{u}} \\
\rightarrow \mathrm{E}_{\mathrm{g}}(32)\end{array}$ & & $\begin{array}{l}\mathrm{Cl}\left(\mathrm{p}_{\mathrm{z}}\right)_{-} \mathrm{d}_{z^{2}} \quad+ \\
\mathrm{A}_{2 \mathrm{u}} \rightarrow \mathrm{E}_{\mathrm{g}} \\
(37)\end{array}$ & $\begin{array}{l}\mathrm{Cl}\left(\mathrm{p}_{\mathrm{x}}\right) \quad \rightarrow \quad \mathrm{d}_{z^{2}} \\
(24)\end{array}$ & $\begin{array}{l}\mathrm{Ph} / \mathrm{E}_{\mathrm{g}} \rightarrow \mathrm{d}_{\mathrm{x}-\mathrm{y}}^{2} \\
(\mathbf{9 3})\end{array}$ \\
\hline $\begin{array}{l}\text { TD-DFT polarizations } \\
\% \mathrm{x} \\
\% \mathrm{y} \\
\% \mathrm{z}\end{array}$ & $\begin{array}{c}0 \\
100 \\
0\end{array}$ & $\begin{array}{c}100 \\
0 \\
0\end{array}$ & $\begin{array}{c}0 \\
0 \\
100 \\
\end{array}$ & $\begin{array}{c}0 \\
100 \\
0\end{array}$ & $\begin{array}{c}100 \\
0 \\
0 \\
\end{array}$ & $\begin{array}{c}0 \\
100 \\
0\end{array}$ \\
\hline
\end{tabular}


Table S6. TD-DFT (B3LYP/LanL2DZ*) results on [ $\left.\mathrm{Mn}^{\mathrm{III}}(\mathrm{TPP}) \mathrm{Cl}\right]$ (Figure 1). Contributions are given in \%.

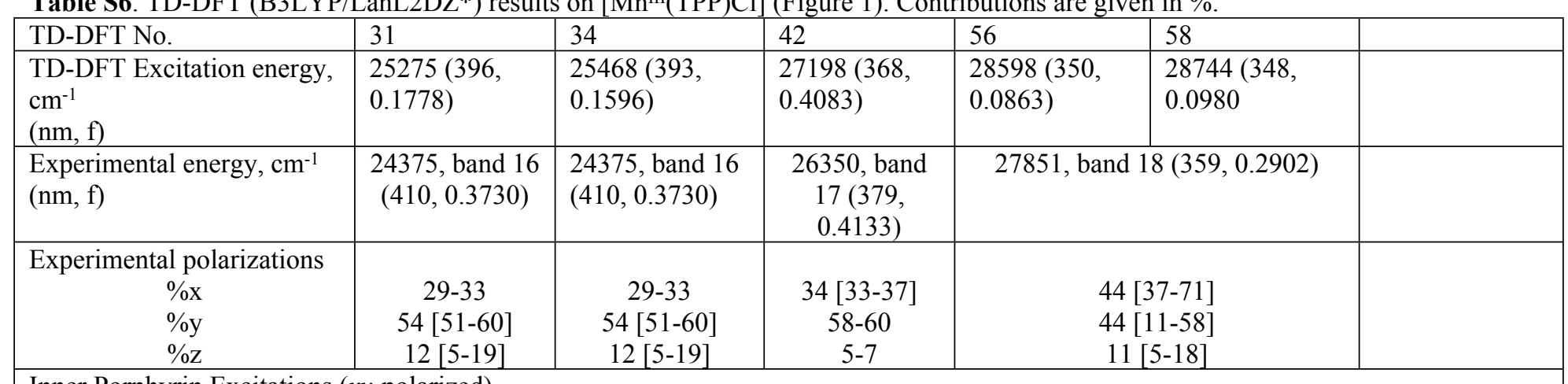

\begin{tabular}{|l|c|c|c|c|c|c|}
\hline Inner Porphyrin Excitations $(x y$-polarized) & 9 & 6 & 11 & & \\
\hline$\pi \rightarrow \pi^{*(0)}$ & & & 6 & 16 & 28 & \\
\hline$\pi \rightarrow \pi^{*(1)}$ & & & & 2 & 1 \\
\hline$\pi \rightarrow \pi^{*(2)}$ & & & & 2 & \\
\hline
\end{tabular}

$\pi \rightarrow \pi^{*(2)}$

\begin{tabular}{|c|c|c|c|c|c|c|}
\hline $\mathrm{CT}^{(1)}$ & 44 & 52 & 48 & & & \\
\hline $\mathrm{CT}^{(2)}$ & 31 & 27 & 18 & & & \\
\hline \multicolumn{7}{|l|}{$\mathrm{CT}^{(3)}$} \\
\hline \multicolumn{7}{|l|}{$\mathrm{CT}^{(4)}$} \\
\hline \multicolumn{7}{|l|}{$\mathrm{CT}^{(5)}$} \\
\hline \multicolumn{7}{|l|}{$\mathrm{CT}^{(6)}$} \\
\hline \multicolumn{7}{|l|}{$\mathrm{CT}^{(7)}$} \\
\hline \multicolumn{7}{|c|}{ Chlorine to Fe Excitations ( $z$-polarized) } \\
\hline \multicolumn{7}{|l|}{$\mathrm{CT}^{(\mathrm{Cl}, \pi)}$} \\
\hline \multicolumn{7}{|l|}{$\mathrm{CT}^{(\mathrm{Cl}, \sigma)}$} \\
\hline Others & $\begin{array}{l}\mathrm{Cl}\left(\mathrm{p}_{\mathrm{z}}\right)_{-} \mathrm{d}_{z^{2}}+ \\
\mathrm{A}_{2 \mathrm{u}} \rightarrow \mathrm{E}_{\mathrm{g}}(16)\end{array}$ & $\begin{array}{l}\mathrm{Cl}\left(\mathrm{p}_{\mathrm{z}}\right)_{-} \mathrm{d}_{z^{2}}+\mathrm{A}_{2 \mathrm{u}} \\
\rightarrow \mathrm{E}_{\mathrm{g}}(14)\end{array}$ & $\begin{array}{l}\mathrm{Cl}\left(\mathrm{p}_{\mathrm{z}}\right)_{-} \mathrm{d}_{z^{2}}+ \\
\mathrm{A}_{2 \mathrm{u}} \rightarrow \mathrm{E}_{\mathrm{g}}(6)\end{array}$ & $\begin{array}{l}\mathrm{Cl}\left(\mathrm{p}_{\mathrm{x}}\right) \rightarrow \mathrm{d}_{z^{2}} \\
(\mathbf{7 5})\end{array}$ & $\begin{array}{l}\mathrm{Cl}\left(\mathrm{p}_{\mathrm{y}}\right) \rightarrow \mathrm{d}_{z^{2}} \\
(\mathbf{6 6})\end{array}$ & \\
\hline $\begin{array}{l}\text { TD-DFT polarizations } \\
\% \mathrm{x} \\
\% \mathrm{y} \\
\% \mathrm{z}\end{array}$ & $\begin{array}{c}0 \\
100 \\
0 \\
\end{array}$ & $\begin{array}{c}100 \\
0 \\
0 \\
\end{array}$ & $\begin{array}{c}100 \\
0 \\
0 \\
\end{array}$ & $\begin{array}{c}100 \\
0 \\
0 \\
\end{array}$ & $\begin{array}{c}0 \\
100 \\
0 \\
\end{array}$ & \\
\hline
\end{tabular}


Table S7. Ground state molecular orbitals in $\left[\mathrm{Mn}^{\mathrm{III}}(\mathrm{TPP}) \mathrm{Cl}\right]$ and $\left[\mathrm{Fe}^{\mathrm{III}}(\mathrm{TPP}) \mathrm{Cl}\right]$ that demonstrate mixing between $\mathrm{A}_{2 \mathrm{u}}<72 / 81>$ and $\mathrm{Cl}\left(\mathrm{p}_{\mathrm{z}}\right) \mathrm{d}_{\mathrm{z}^{2}}$ and/or $\mathrm{d}_{\mathrm{z}^{2}}$.

\begin{tabular}{|l|l|l|l|}
\hline \multicolumn{2}{|c|}{$\left[\mathrm{Mn}^{\mathrm{III}}(\mathrm{TPP}) \mathrm{Cl}\right]$} & \multicolumn{2}{c|}{$\left[\mathrm{Fe}^{\mathrm{III}}(\mathrm{TPP}) \mathrm{Cl}\right]^{a}$} \\
\hline MOs & $\%$ Contribution & MOs & $\%$ Contribution \\
\hline$\alpha<\mathbf{1 7 3}>\mathrm{HOMO}$ & $7 \% \mathrm{Mn}, 10 \% \mathrm{Cl}, 71 \%$ Por & & \\
\hline$\alpha<\mathbf{1 7 1}>$ & $19 \% \mathrm{Mn}, 39 \% \mathrm{Cl}, 30 \%$ Por & & \\
\hline$\alpha<\mathbf{1 5 7}>$ & $2 \% \mathrm{Mn}, 12 \% \mathrm{Cl}, 66 \%$ Por & & $9 \% \mathrm{Fe}, 2 \% \mathrm{Cl}, 87 \%$ Por \\
\hline$\beta<\mathbf{1 6 9}>\mathrm{HOMO}$ & $2 \% \mathrm{Mn}, 79 \%$ Por & $\beta<169>\mathrm{HOMO}$ & \\
\hline$\beta<\mathbf{1 5 8}>$ & $4 \% \mathrm{Mn}, 9 \% \mathrm{Cl}, 19 \%$ Por & & $4 \% \mathrm{Fe}, 94 \%$ Por \\
\hline$\beta<\mathbf{1 5 6}>$ & $8 \% \mathrm{Mn}, 25 \% \mathrm{Cl}, 34 \%$ Por & $\beta<156>$ & \\
\hline$\beta<\mathbf{1 5 3}>$ & $4 \% \mathrm{Mn}, 37 \% \mathrm{Cl}, 41 \%$ Por & & \\
\hline
\end{tabular}

${ }^{a}$ Ref. 6.

Table S8. Electronic transitions present in bands 10-18 that originate from the mixed MOs listed in Tables 1 and 2 (based on the TD-DFT results).

\begin{tabular}{|c|c|c|c|c|c|c|c|c|c|}
\hline \multirow[t]{2}{*}{ Transitions } & \multicolumn{9}{|c|}{ Band number } \\
\hline & 10 & 11 & 12 & 13 & 14 & 15 & 16 & 17 & 18 \\
\hline \multicolumn{10}{|l|}{$\alpha$ Molecular Orbitals } \\
\hline$\alpha<\mathbf{1 7 3}>\rightarrow \alpha<175 / 176>\left(\pi \rightarrow \pi^{*(0)}\right)$ & $\mathrm{xxx}$ & $\mathrm{xxx}$ & & & & & $\mathrm{xxx}$ & $\mathrm{xxx}$ & \\
\hline$\alpha<\mathbf{1 7 1}>\rightarrow \alpha<175 / 176>(\mathrm{CT})$ & $\mathrm{xxx}$ & $\mathrm{xxx}$ & & $\mathrm{xxx}$ & $\mathrm{xxx}$ & & $\mathrm{xxx}$ & $\mathrm{xxx}$ & \\
\hline \multicolumn{10}{|l|}{$\beta$ Molecular Orbitals } \\
\hline$\beta<\mathbf{1 6 9}>\rightarrow \beta<170 / 171>\left(\pi \rightarrow \pi^{*(0)}\right)$ & & $\mathrm{xxx}$ & & & & & $\mathrm{xxx}$ & $\mathrm{xxx}$ & \\
\hline$\beta<\mathbf{1 5 6}>\rightarrow \beta<170 / 171>\left(\pi \rightarrow \pi^{*(1)}\right)$ & & & & $\mathrm{xxx}$ & $\mathrm{xxx}$ & $\mathrm{xxx}$ & & $\mathrm{xxx}$ & $\mathrm{xxx}$ \\
\hline$\beta<\mathbf{1 5 8}>\rightarrow \beta<170 / 171>\left(\pi \rightarrow \pi^{*(1)}\right)$ & & & & $\mathrm{xxx}$ & $\mathrm{xxx}$ & $\mathrm{xxx}$ & & & $\mathrm{xxx}$ \\
\hline$\beta<\mathbf{1 5 3}>\rightarrow \beta<170>\left(\mathrm{Cl}\left(\mathrm{p}_{\mathrm{z}}\right)_{-} \mathrm{d}_{\mathrm{z}}^{2}+\mathrm{A}_{2 \mathrm{u}} \rightarrow \mathrm{E}_{\mathrm{g}}\right)$ & & & & & & $\mathrm{xxx}$ & & $\mathrm{xxx}$ & \\
\hline$\beta<153>\rightarrow \beta<173 / 175>\left(\mathrm{CT}^{\mathrm{Cl}, \sigma}\right)$ & & & $\mathrm{xxx}$ & & & $\mathrm{xxx}$ & & & \\
\hline$\beta<\mathbf{1 5 6}>\rightarrow \beta<175>\left(\mathrm{CT}^{1}\right)$ & & & $\mathrm{xxx}$ & & & & & & \\
\hline$\beta<\mathbf{1 6 9}>\rightarrow \beta<173 / 174>\left(\mathrm{CT}^{2}\right)$ & $\mathrm{xxx}$ & $\mathrm{xxx}$ & & $\mathrm{xxx}$ & $\mathrm{Xxx}$ & $\mathrm{xxx}$ & $\mathrm{xxx}$ & $\mathrm{xxx}$ & \\
\hline$\beta<\mathbf{1 5 6}>\rightarrow \beta<173 / 174>\left(\mathrm{CT}^{4}\right)$ & & & & & $\mathrm{xxx}$ & $\mathrm{xxx}$ & & & \\
\hline$\beta<\mathbf{1 5 8}>\rightarrow \beta<173 / 174>\left(\mathrm{CT}^{4}\right)$ & & & & $\mathrm{xxx}$ & $\mathrm{xxx}$ & $\mathrm{xxx}$ & & & \\
\hline
\end{tabular}

${ }^{a}$ The black squares inside the box indicate that the electronic transition is present within the corresponding band. 


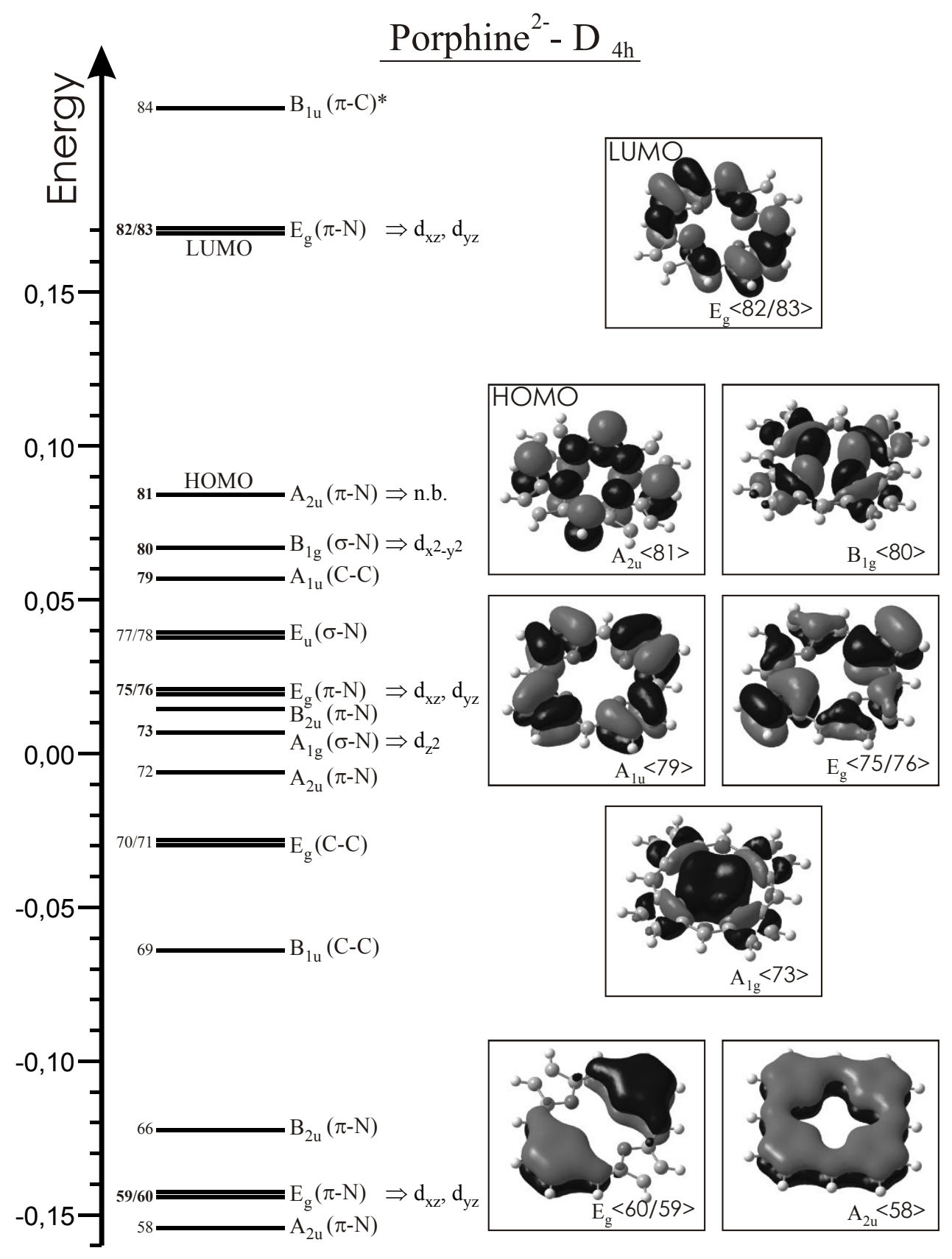

Figure S1. Molecular orbital (MO) diagram of the free porphine ${ }^{2-}$ ligand and some contour plots. Energies are given in Hartree. Metal d orbitals that potentially interact with these MOs are indicated (in $\mathrm{D}_{4 \mathrm{~h}}$ symmetry). 

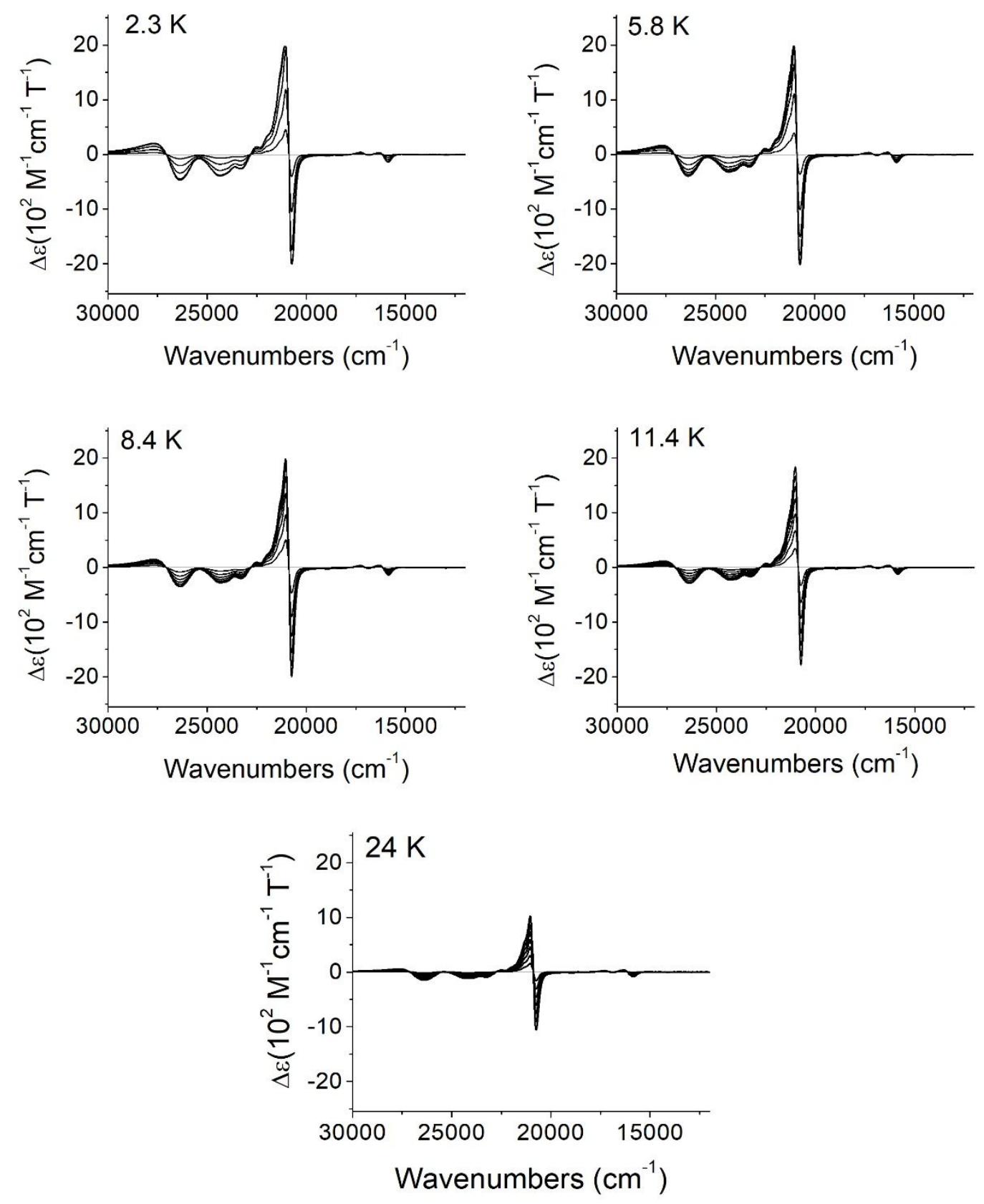

Figure S2. MCD spectra of $\left[\mathrm{Mn}^{\mathrm{III}}(\mathrm{TPP}) \mathrm{Cl}\right]$ taken at various temperatures and various magnetic fields. 


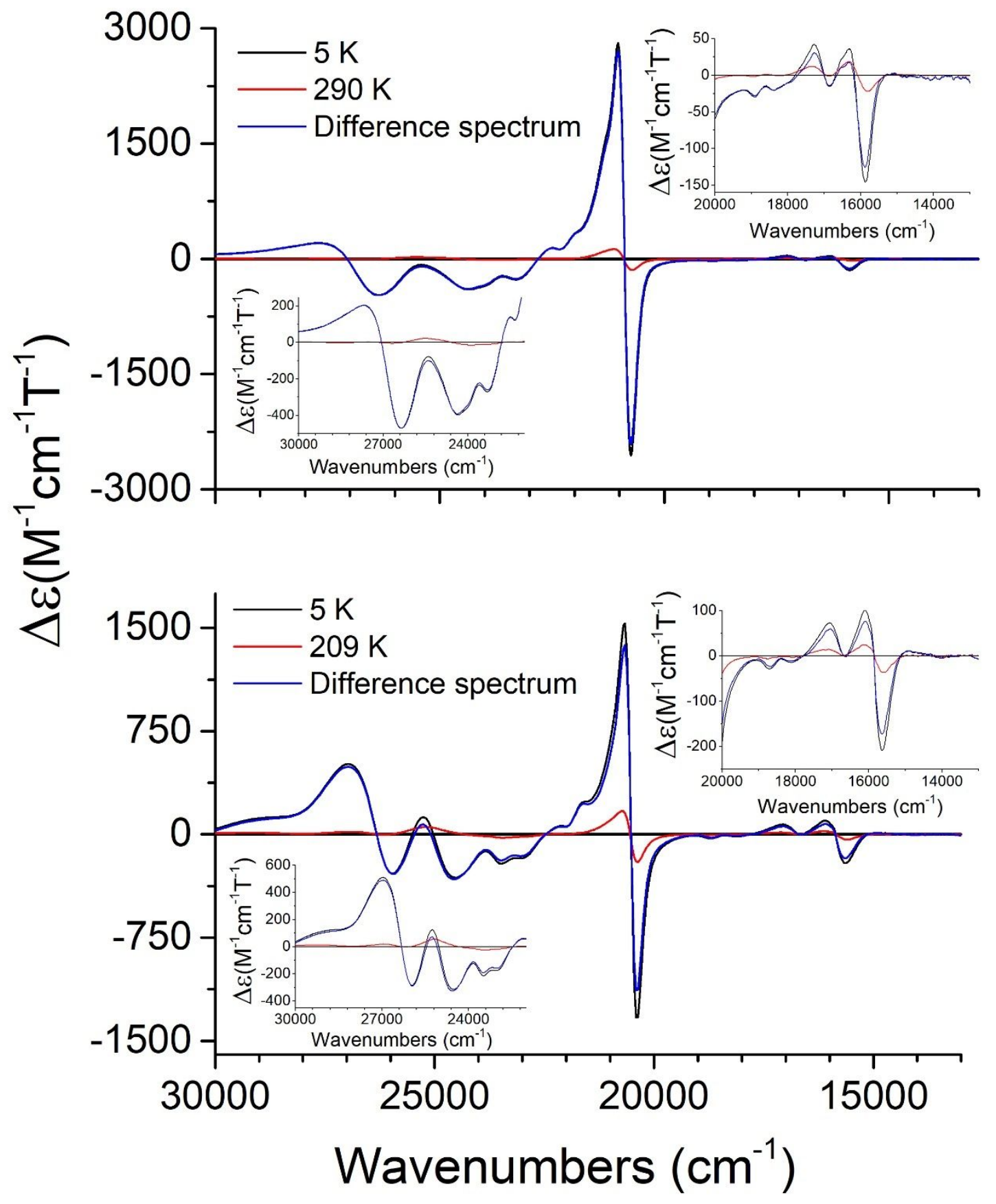

Figure S3. MCD spectra of $\left[\mathrm{Mn}^{\mathrm{III}}(\mathrm{TPP}) \mathrm{Cl}\right]$ (top) and [Mn $\left.{ }^{\mathrm{III}}(\mathrm{TPP}) \mathrm{Br}\right]$ (bottom): The black line represents the MCD spectrum measured at $5 \mathrm{~K}$, and the red line depicts the spectrum taken at $290 \mathrm{~K}$ (for $\left[\mathrm{Mn}^{\mathrm{III}}(\mathrm{TPP}) \mathrm{Cl}\right]$ ) and $209 \mathrm{~K}$ (for $\left[\mathrm{Mn}^{\mathrm{III}}(\mathrm{TPP}) \mathrm{Br}\right]$ ). The $\mathbf{C}$-term spectrum in blue results from the subtraction of the red spectrum from the black one. 

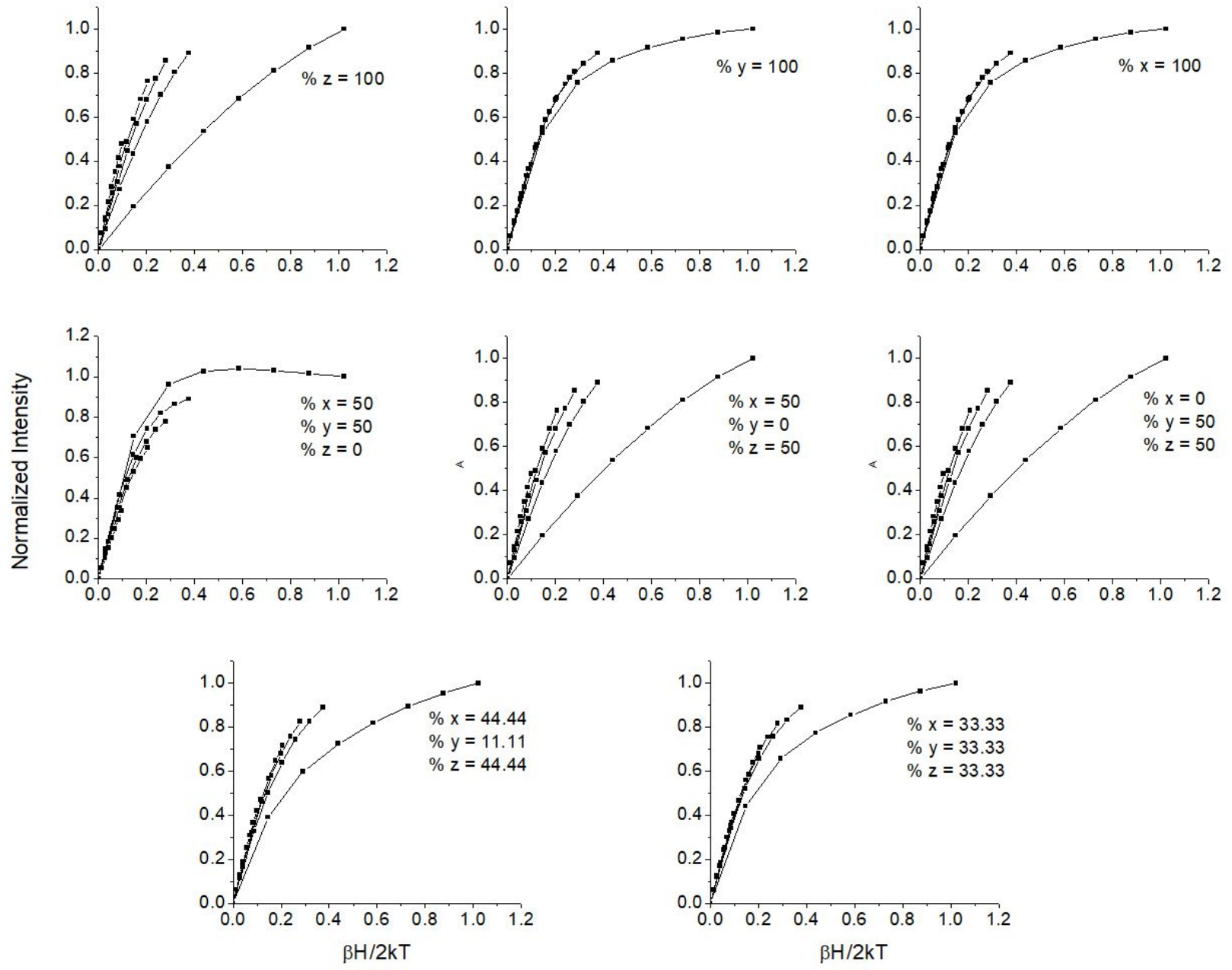

Figure S4. Calibration of VTVH curves using $\mathrm{S}=2, \mathrm{E} / \mathrm{D}=0 ; \mathrm{D}=-2.29 \mathrm{~cm}^{-1}$; and $\mathrm{g}_{\mathrm{x}}, \mathrm{g}_{\mathrm{y}}, \mathrm{g}_{\mathrm{z}}=2.0023$. The isotherms were calculated at $2.3,5.8,8.4,11.4$, and $24.0 \mathrm{~K}$. 

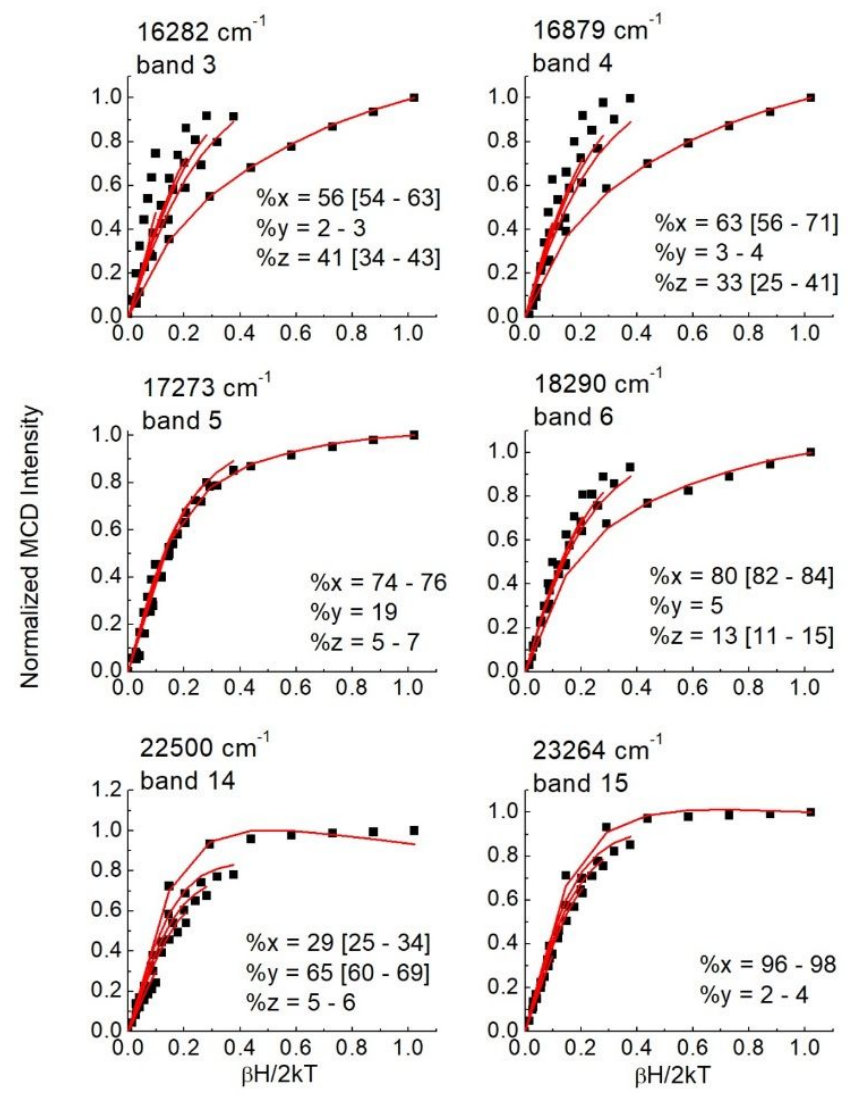

Figure S5. MCD C-term magnetic saturation curves (VTVH) of [ $\left.\mathrm{Mn}^{\mathrm{III}}(\mathrm{TPP}) \mathrm{Cl}\right]$ at different (selected) energies, showing the polarizations of the different optical transitions. The red lines pertain to the best fit of the experimental data (black dots). The fits for bands 3 and 4 are not very good, due to the low MCD intensity of these features, which induces large errors into the experimental data.

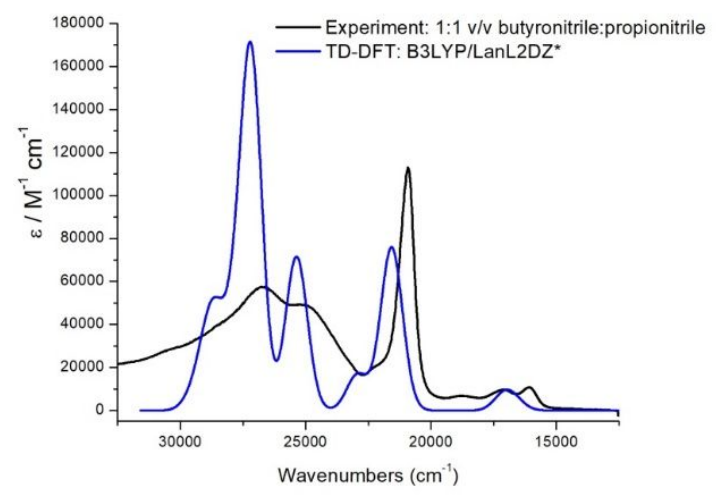

Figure S6. TD-DFT-derived (blue) and experimental (black) absorption spectrum of $\left[\mathrm{Mn}{ }^{\mathrm{III}}(\mathrm{TPP}) \mathrm{Cl}\right]$ in $1: 1 \mathrm{v} / \mathrm{v}$ butyronitrile:propionitrile mixture. 

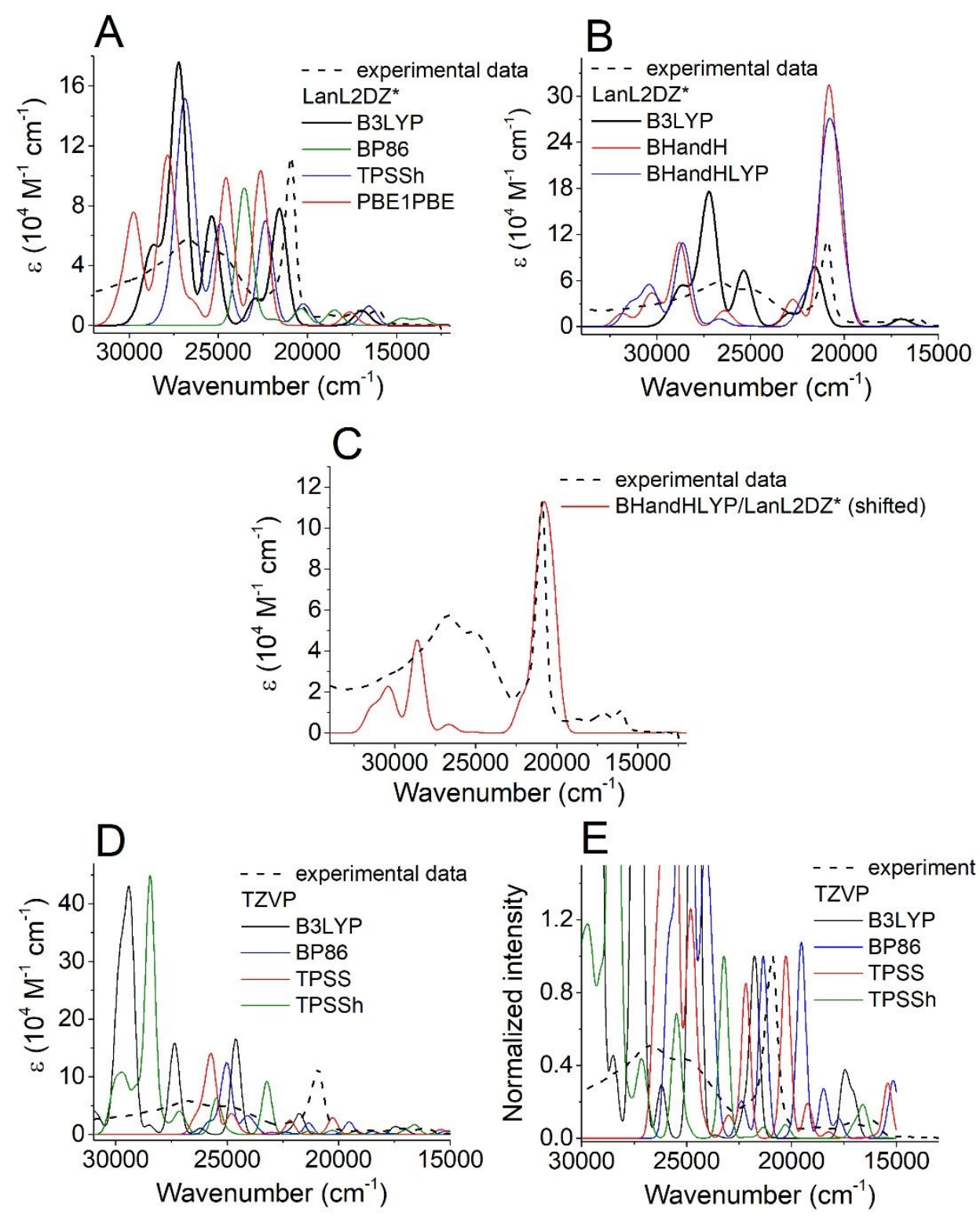

Figure S7. TD-DFT spectra of $\left[\mathrm{Mn}^{\mathrm{III}}(\mathrm{TPP}) \mathrm{Cl}\right]$ derived using different functionals with basis set LanL2DZ* (plots A-C), and TZVP (plots D and E). Plots A and B show the actual intensities of the theoretical spectra in relation to the experimental data. The theoretical plots generated by BHandH and BHandHLYP were shifted to lower energy by $5000 \mathrm{~cm}^{-1}$. Plot $\mathrm{C}$ shows an overlay of the experimental and BHandHLYP-derived data normalized to the Soret band intensity, with the theoretical spectrum shifted to lower energy by $5000 \mathrm{~cm}^{-1}$. Plot E shows a set of spectra with their Soret band intensities normalized to the experimentally observed Soret band intensity. The theoretical spectra were generated using a FWHM of $485 \mathrm{~cm}^{-1}$. The experimental spectrum was obtained in $1: 1 \mathrm{v} / \mathrm{v}$ butyronitrile:propionitrile. 

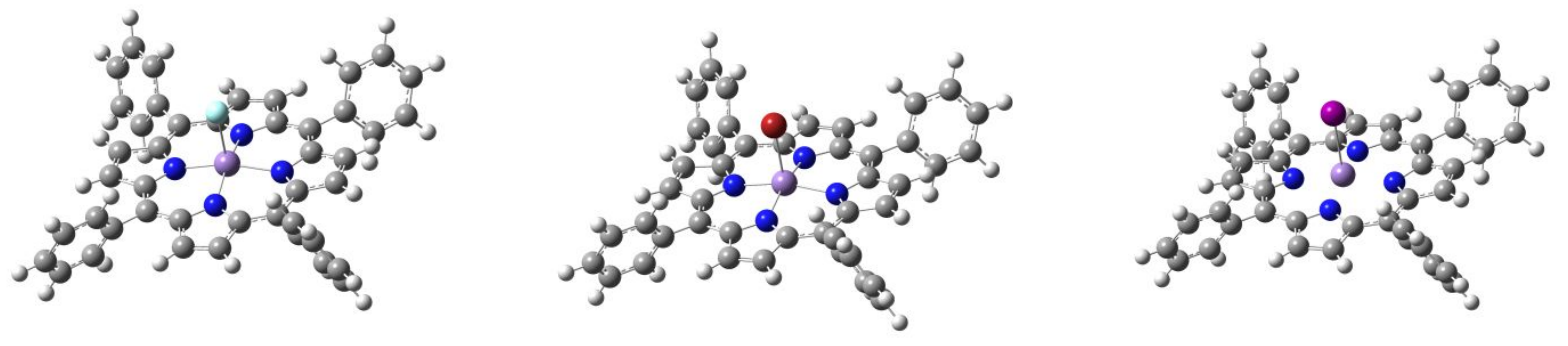

Figure S8. Optimized structures of $\left[\mathrm{Mn}^{\mathrm{III}}(\mathrm{TPP}) \mathrm{F}\right]$ (left), [Mn $\left.{ }^{\mathrm{III}}(\mathrm{TPP}) \mathrm{Br}\right]$ (middle) and [Mn $\left.{ }^{\mathrm{III}}(\mathrm{TPP}) \mathrm{I}\right]$ (right). The light cyan, red, magenta, purple, and blue spheres represent the fluoride, bromide, iodide, $\mathrm{Mn}^{\mathrm{III}}$, and pyrrole nitrogens, respectively.
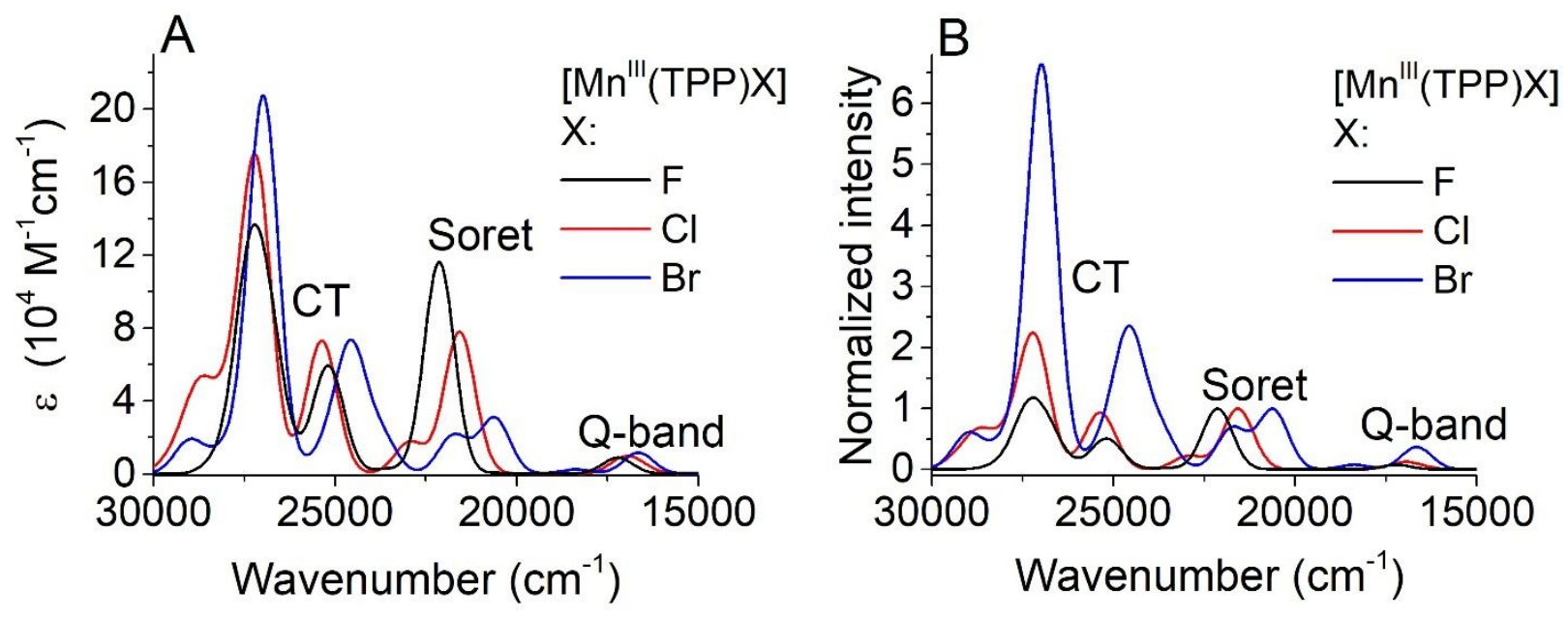

Figure S9. TD-DFT derived spectra of the $\left[\mathrm{Mn}^{\mathrm{III}}(\mathrm{TPP}) \mathrm{X}\right]$ complexes using B3LYP/LanL2DZ*. Plot A shows the actual intensities of the spectral features, while Plot B shows the data normalized to the Soret band intensity. 


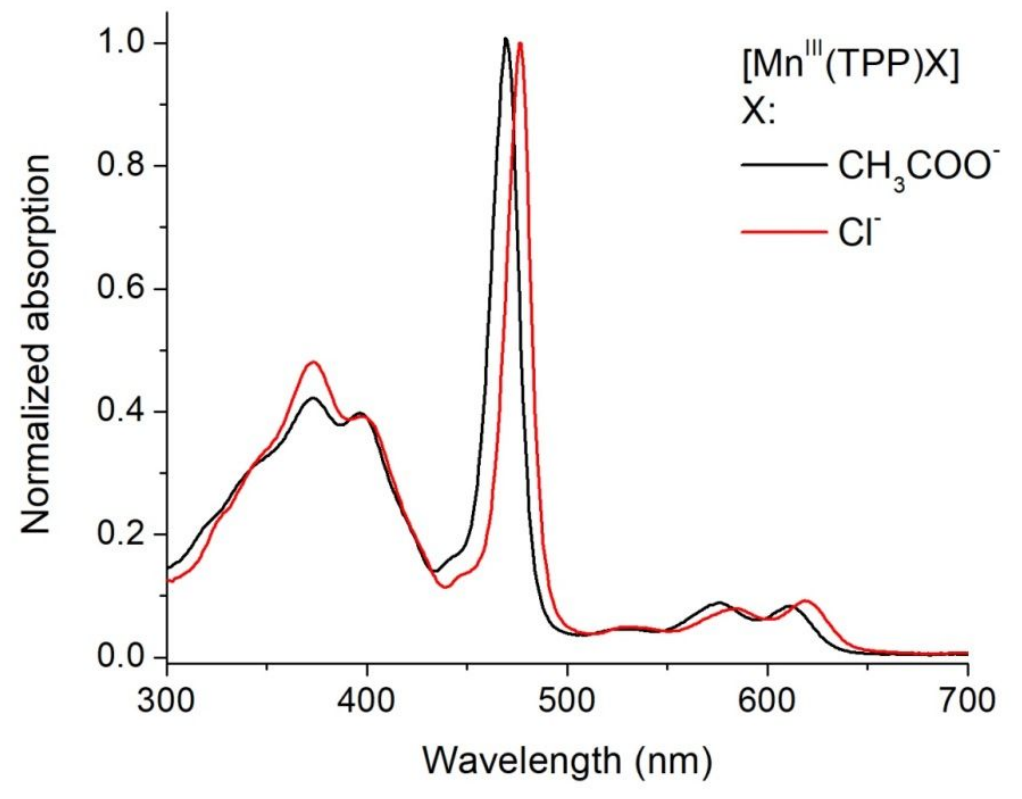

Figure S10. Absorption spectra of two $\left[\mathrm{Mn}^{\mathrm{III}}(\mathrm{TPP}) \mathrm{X}\right]$ complexes in dichloromethane.

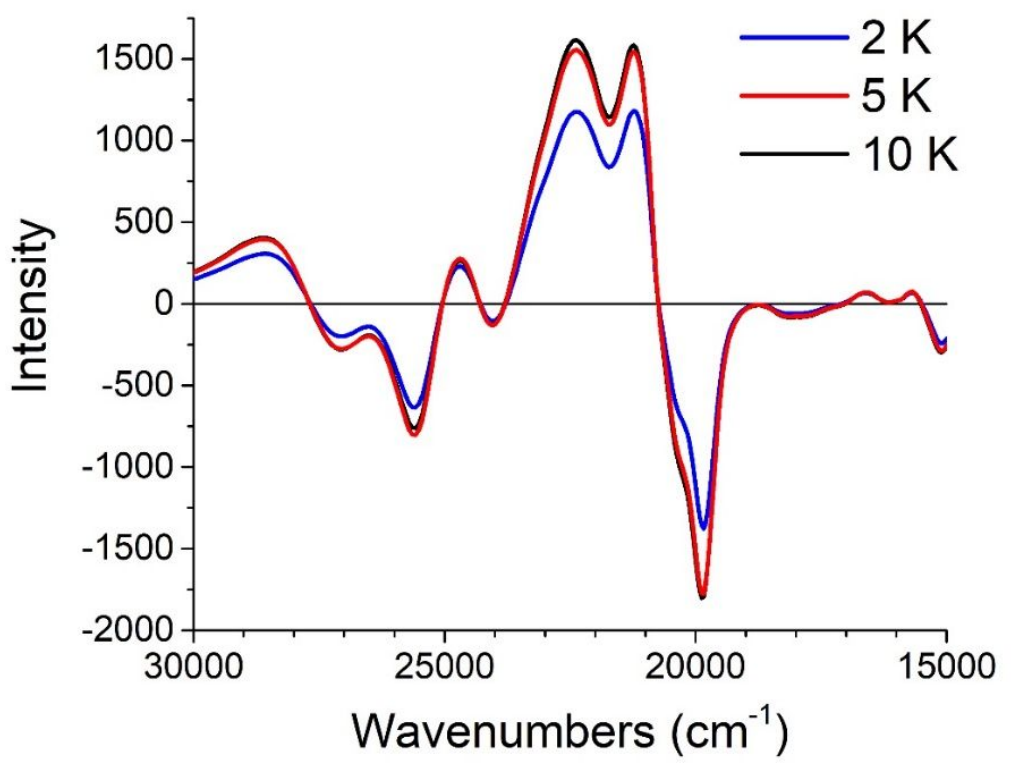

Figure S11. MCD spectra of [ $\left.\mathrm{Mn}^{\mathrm{III}}(\mathrm{TPP}) \mathrm{I}\right]$ obtained at different temperatures at $7 \mathrm{~T}$. Unlike the chloro and bromo complex that displays a clean pseudo A-type signal in the Soret region (Figure 10), the iodo analogue shows an intense positive feature at $22380 \mathrm{~cm}^{-1}$, which comes from an impurity. 


\section{References:}

1. Powell, M. F.; Pai, E. F.; Bruice, T. C. Study of (tetraphenylporhinato)manganese(III)-catalyzed epoxidation and demethylation using p-cyano-N,N-dimethylaniline $\mathrm{N}$-oxide as oxygen donor in a homogenous system: Kinetics, radiochemical ligation studies, and reaction mechanism for a model of cytochrome P-450. J. Am. Chem. Soc. 1984, 106, 3277-3285.

2. Turner, P.; Gunter, M. J. Carbon-13 NMR spectroscopy, electron spin distributions, and valence state of pentacoordinate manganese tetraphenyl complexes. Inorg. Chem. 1994, 33, 1406-1415.

3. Paulat, F.; Praneeth, V. K. K.; Näther, C.; Lehnert, N. Quantum chemistry-based analysis of the vibrational spectra of five-coordinate metalloporphyrins [M(TPP)Cl]. Inorg. Chem. 2006, 45, 2835-2856.

4. $\quad$ Armstrong, R. S.; Foran, G. J.; Hambley, T. W. Structure of chloro(a,b,g,dtetraphenylporphinato)manganese(III) toluene solvate. Acta Crystallogr. C 1993, 49, 236-238.

5. Turner, P.; Gunter, M. J.; Skelton, B. W.; White, A. H. The influence of the axial ligand on the solid-state structures of pentacoordinate manganese tetraphenylporphyrin complexes. Aust. J. Chem. 1998, 51, 853-863.

6. Paulat, F.; Lehnert, N. Detailed assignment of the magnetic circular dichroism and UV-vis spectra of five-coordinate high-spin ferric [Fe(TPP)(Cl)]. Inorg. Chem. 2008, 47, 4963-4976. 